\title{
The T $\alpha 1 \alpha$-Tubulin Promoter Specifies Gene Expression as a Function of Neuronal Growth and Regeneration in Transgenic Mice
}

\author{
A. Gloster, ${ }^{1}$ W. Wu, ${ }^{1}$ A. Speelman, ${ }^{1}$ S. Weiss, ${ }^{2}$ C. Causing, ${ }^{1}$ C. Pozniak, ${ }^{1}$ B. Reynolds, ${ }^{2}$ E. Chang, ${ }^{1}$ J. G. Toma, ${ }^{3}$ \\ and F. D. Miller ${ }^{1}$ \\ 'Center for Neuronal Survival and Department of Neurology and Neurosurgery, Montreal Neurological Institute, McGill \\ University, Montreal, Quebec, Canada H3A 2B4, ${ }^{2}$ Department of Anatomy, University of Calgary, Health Sciences Center, \\ Calgary, Alberta, Canada T2N 4N1, and ${ }^{3}$ Department of Anatomy and Cell Biology, University of Alberta, Edmonton, \\ Alberta, Canada T6G 2H7
}

\begin{abstract}
We have previously demonstrated that one member of the $\alpha$-tubulin multigene family, termed $T \alpha 1$ in rats, is regulated as a function of neuronal growth and regeneration. To elucidate the molecular mechanisms responsible for coupling gene expression to morphological differentiation, we have isolated the $T \alpha 1$ gene, have fused $1.1 \mathrm{~kb}$ of the $5^{\prime}$ flanking region to a nuclear lac $Z$ reporter gene, and have generated transgenic mice. Analysis of these transgenic mice demonstrated that marker gene expression was specific to the CNS and PNS, with expression in vivo at embryonic day 13.5 being similar to expression of the endogenous gene. Moreover, the induction of transgene expression was correlated temporally with neuronal commitment in developing neural crest-derived peripheral neurons and in the developing retina. Immunocytochemical analysis of mixed primary embryonic brain cultures confirmed that transgene expression was specific to neurons, with the majority of neurons, but not astrocytes or oligodendrocytes, expressing $\beta$-galactosidase. Transgene expression in vivo was maintained in developing neurons until early in postnatal life, subsequent to which its expression decreased coincident with neuronal maturation. The transgene was then reinduced in regenerating facial motoneurons following unilateral axotomy of the facial nerve. Thus, $1.1 \mathrm{~kb}$ of $5^{\prime}$ flanking sequence from the $T_{\alpha 1}$ gene contains the sequence elements responsible for specifying gene expression to embryonic neurons and for subsequently regulating gene expression in both developing and mature neurons as a function of morphological growth.

[Key words: neuronal development, neuronal regeneration, neuronal growth, $\alpha$-tubulin, neuronal promoters, transgenic mice]
\end{abstract}

\footnotetext{
Received Feb. 16, 1994; revised May 13, 1994; accepted June 15, 1994.

We thank our colleagues Drs. R. Campenot and W. Gallin for all of their thoughtful advice throughout the course of this work, D. Senger for her help with the Western blots, and R. Varma, L.-J. Duan, L. Mah, and J. Hewson for invaluable technical assistance. We also thank John Roder, Susan Clapoff, and the NCE for Neural Regeneration supplementary core facility for generating the transgenic moluse lines used in this study. This work was funded by grants fram the IRC and the NeuroScience Network to F.D.M. and S.W. S.W. is an MRC cientist and AHFMR Scholar. C.C.,E., A.G., and W.W. are supported by the Savoy Foundation, the AHFMR, the NeuroScience Network, and the Rick Hansen Foundation for Spinal Cord Research, respectively.

Correspondence should be addressed to F. D. Miller, Center for Neuronal Survival, Fieldhouse Laboratories, Montreal Neurological Institute, 3801 rue University, Montreal, Quebec, Canada H3A 2B4.

Copyright (C) 1994 Society for Neuroscience $0270-6474 / 94 / 147319-12 \$ 05.00 / 0$
}

Microtubules, which are assembled from $\alpha$ - and $\beta$-tubulins, comprise the major cytoskeletal component of growing neurites (Daniels, 1972). In mammals, at least six different $\alpha$-tubulin genes (Villasante et al., 1986) and five different $\beta$-tubulin genes (Wang et al., 1986) are expressed in neural and non-neural tissues at various times during development. We have previously demonstrated that, of two $\alpha$-tubulin genes known to be expressed in the embryonic nervous system of the rat, one, termed $T \alpha 1$, is abundantly expressed in developing neurons during morphological growth, while a second, termed T26, is constitutively expressed in neurons and non-neuronal cells (Miller et al., 1987). Moreover, it is likely that the T $\alpha 1$ isotype is incorporated in the majority of neuronal microtubules during development, since T $\alpha 1$ mRNA comprises $1-2 \%$ of the total mRNA, and $>95 \%$ of the total $\alpha$-tubulin mRNA in the embryonic nervous system.

Expression of T $\alpha 1 \alpha$-tubulin mRNA is also correlated with the growth of mature neurons. Following axotomy of motor (Miller et al., 1989), and sympathetic (Mathew and Miller, 1990) neurons, T $\alpha 1$ mRNA increases rapidly, and then decreases to control levels following target reinnervation. If regeneration is unsuccessful (Miller et al., 1989), as with CNS neurons (Tetzlaff et al., 1991), T $\alpha 1$ mRNA levels remain elevated. These increases appear to be due, to a great extent, to loss of repressive homeostatic signals arising from the nonterminal axon (Mathew and Miller, 1993; Wu et al., 1993). T $\alpha 1 \alpha$-tubulin mRNA levels are also increased during collateral sprouting of adult sympathetic neurons (Mathew and Miller, 1990), presumably in response to increased target-derived NGF, since exogenous NGF can increase T $\alpha 1 \alpha$-tubulin mRNA levels in these neurons both in vivo (Mathew and Miller, 1990; Miller et al., 1994) and in culture (Ma et al., 1992). Thus, expression of T $\alpha 1$ mRNA is high during developmental growth, is downregulated as a function of neuronal maturation, and is then increased in response to extrinsic cues that regulate the growth of mature neurons.

The functional role of these high levels of expression of one particular $\alpha$-tubulin isotype during neuronal growth remains speculative. The rat T $\alpha 1$ mRNA is homologous to the human $\mathrm{b} \alpha 1$, and mouse M $\alpha 1$ mRNAs (Lemischka et al., 1981; Cowan et 2l., 1983. Lewis ef al, 1985) whereas the rat T26 MRRA (Ginzburg et al., 1986) is homologous to the human kal (Cowan et al., 1983), and mouse $\mathrm{M} \alpha 2$ (Lewis et al., 1985) mRNAs. The $\mathrm{T} \alpha 1 / \mathrm{b} \alpha 1 / \mathrm{M} \alpha 1 \alpha$-tubulin isotype differs from the T26/k $\alpha 1 / \mathrm{M} \alpha 2$ isotype by virtue of a single amino acid difference (glycine to serine) at residue 232 . We have therefore previously speculated that T $\alpha 1$ and T26 mRNAs encode functionally equivalent pro- 
teins, but that the $T \alpha 1$ gene itself is specialized to produce a large pool of $\alpha$-tubulin monomers when this is in high demand for neurite extension.

To define the molecular genetic mechanisms responsible for coupling gene expression to neuronal growth, we have isolated the putative promoter region of the $\mathrm{T} \alpha 1$ gene, and in this article we report that $1.1 \mathrm{~kb}$ of this promoter region is sufficient to regulate gene expression as a function of the growth of developing and regenerating neurons in transgenic mice.

\section{Materials and Methods}

Library screening. A Wistar rat $\lambda$-DASH genomic library purchased from Stratagene was plated on a lawn of Escherichia coli strain LE392. Plaque lifts were made onto nitrocellulose filters, which were probed with the end-labeled oligomers 5'-GGCAGGTTCTCTTACATCGA, and $5 '$-CTCTGGGCAGACGGAGGGGT, which were derived from the $5^{\prime}$ untranslated region and the immediately $5^{\prime}$ upstream flanking region of the previously published sequence of the T $\alpha 1 \alpha$-tubulin gene (Lemischka and Sharp, 1982). Two identical lambda clones were identified.

Construction of the $p T \alpha 1: n l a c Z$ transgene and transgenic mice. A 1.9 $\mathrm{kb}$ PstI fragment containing $1.6 \mathrm{~kb}$ of $5^{\prime}$ genomic flanking sequences, the region corresponding to the $5^{\prime}$ untranslated sequences, the translation start site (exon 1), and 200 nucleotides of intervening sequences (intron 2) was subcloned into the PstI site of pGEM-4Z. An SstI/NcoI partial digestion product that contained 1028 nucleotides of 5 ' flanking sequence, the $5^{\prime}$ untranslated region (99 nucleotides), and the ATG translational start site was isolated. We then cut the plasmid pMaori3 with SstI and NcoI (which cuts at the translational start site of the nlacZ gene in this plasmid), and ligated in the T $\alpha 1 \mathrm{SstI} / \mathrm{NcoI}$ fragment, thereby replacing the $\mathrm{CMV}$ promoter with the T $\alpha 15^{\prime}$ flanking region. pMaori3, which is a derivative of pnlacF (Mercer et al., 1991), was kindly provided by Dr. Eric Mercer. This plasmid contains a CMV promoter linked to a lacZ gene that has had a nuclear translocation signal (Landford et al., 1988) inserted in frame with the lac $Z$ coding sequence to encode a nuclear-targeted version of $E$. coli $\beta$-galactosidase. pMaori3 also includes, downstream to the lacZ gene, the murine protamine-1 gene (Peschon et al., 1987) from +95 relative to the transcription start site to +625 , which provides an intron and a polyadenylation signal. The T $\alpha 1$ :nlacZ transgene was then excised from this plasmid using Sall and EcoRI, and subcloned into the same two sites in the pUC19 polylinker. The transgene fragment was isolated free of vector sequences from this plasmid by digestion with HindIII and XbaI. Sequencing of the T $\alpha 1$ promoter fragment was performed using the Sanger dideoxy method (Sanger et al., 1977), thereby obtaining completely overlapping sequence information in both directions.

Transgenic mice were generated by the Networks of Centers of Excellence for Neural Regeneration and Recovery transgenic core facility. Presence of the transgene was identified by tail blots. Animals from eight $T \alpha 1$ :nlacZ lines were identified, five of which showed expression.

Animals and surgical procedures. For developmental studies, transgenic CD1 mice were bred, and the morning of the plug was counted as day 0.5 . Whole embryos were fixed and stained as described below.

For regeneration studies, adult male or female transgenic mice were anesthetized with sodium pentobarbital $(35 \mathrm{mg} / \mathrm{kg}$ ), and the main branch of the facial nerve was transected or crushed as it exited the stylomastoid foramen, taking care not to injure the adjacent blood vessels. Five days following axotomy, animals were anesthetized with sodium pentobarbital and transcardially perfused with $4 \%$ paraformaldehyde. The mouse brains were then dissected, and sliced into sections 1-2 mm thick prior to X-gal staining. In total, nine animals were examined, with five undergoing a unilateral transection, and four undergoing a unilateral crush. No difference in response was noted between male and female mice.

$L a c Z$ staining technique. Embryos and brains were prepared for $E$. coli $\beta$-galactosidase staining by fixing for $60 \mathrm{~min}$ at $4^{\circ} \mathrm{C}$ in fresh $4 \%$ paraformaldehyde, $0.1 \mathrm{M} \mathrm{NaH} \mathrm{PO}_{4}(\mathrm{pH}$ 7.3). They were then rinsed three times, for $30 \mathrm{~min}$ each, with a wash containing $0.1 \mathrm{M} \mathrm{NaH}_{2} \mathrm{PO}_{4}$ (pH 7.3), 2 mM $\mathrm{MgCl}_{2}, 0.01 \%$ sodium deoxycholate, and $0.02 \% \mathrm{NP}-$ 40 . The staining reaction was performed by incubating the tissue at $37^{\circ} \mathrm{C}$ in a reaction mix containing all the components of the rinse buffer with

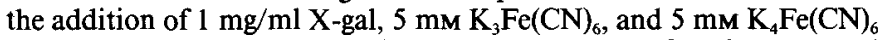
(at pH 7.3-7.6). For the Q54 line, staining was done for $12-24 \mathrm{hr}$, and for the $\mathrm{K} 6$ line, for 3-5 hr. For embryos, a postfixation was performed for $24-48 \mathrm{hr}$ in $4 \%$ paraformaldehyde. Brain slices were instead left in rinse buffer for a similar time period. Embryos and brain slices to be used for sectioning were cryoprotected in graded sucrose solutions, as we have previously described (Miller et al., 1987), and sectioned on the cryostat.

Western blot analysis. Frozen tissues were crushed and homogenized in lysis buffer ( $20 \mathrm{~mm}$ Tris, $1 \%$ Triton-X, 5 mM EDTA, $100 \mathrm{~mm}$ PMSF, 1:1000 leupeptin, 1:1000 aprotinin) by passing the homogenate through a 22 gauge needle. Protein concentrations were determined using a bicinchoninic acid protein assay (Sigma). Proteins were separated on an $8 \%$ SDS polyacrylamide gel. The gel was rinsed in Towbin buffer, and the proteins transferred to nitrocellulose using a Hoefer semidry transfer unit. The nitrocellulose was blocked for $1 \mathrm{hr}$ with $5 \%$ dried milk in PBS with $0.3 \%$ Tween, washed three times in PBS with $0.1 \%$ Tween, and incubated with 1:5000 rabbit anti- $\beta$-galactosidase for $1 \mathrm{hr}$ (Cappel, Durham, NC). The filters were subsequently washed three times with PBS plus $0.1 \%$ Tween, and incubated with a 1:5000 dilution of peroxidase-conjugated goat anti-rabbit antibody for $1 \mathrm{hr}$ (Jackson Labs). The ECL system was used to visualize reactive proteins (Amersham).

In situ hybridization. In situ hybridization on E16 rat embryos was performed as we have previously described (Miller et al., 1987). Briefly, embryos were isolated, immersion fixed in $4 \%$ paraformaldehyde overnight, cryoprotected through graded sucrose solutions $(12 \%, 16 \%$ and $18 \%$ ), frozen, and sectioned onto chrom-alum-subbed slides. Antisense ${ }^{35} \mathrm{~S}$-RNA probes specific for T $\alpha 1 \alpha$-tubulin mRNA were generated and hybridized to these sections as described (Miller et al., 1987, 1989). Slides were then dipped in Kodak NTB-3 emulsion, exposed for 5-10 $\mathrm{d}$, developed, and analyzed using dark-field microscopy.

Primary neuronal cultures. Whole brains from E14 $\mathrm{T}^{\alpha} \alpha 1 \alpha$-tubulin CD1 transgenic mice (bred and maintained by the transgenic facility at the University of Alberta) were stored overnight in $30 \mathrm{~mm} \mathrm{KCl}, 5 \mathrm{~mm}$ glucose, $0.24 \mathrm{mM} \mathrm{MgCl}_{2}, 10 \mathrm{~mm} \mathrm{NaH} \mathrm{PO}_{4}, 5 \mathrm{mM} \mathrm{Na}_{2} \mathrm{HPO}_{4}, 20 \mathrm{~mm}$ lactic acid, and $140 \mathrm{~mm}$ sorbitol, and then were mechanically dissociated with a fire-polished Pasteur pipette in serum-free medium composed of 1:1 mixture of Dulbecco's modified Eagle's medium (DMEM) and F-12 nutrient (GIBCO), including glucose (0.6\%), glutamine (2 mM), sodium bicarbonate ( $3 \mathrm{~mm}$ ), HEPES buffer $(5 \mathrm{~mm}$ ) (all from Sigma except glutamine, from GIBCO), and a defined hormone mixture. Cells $\left(0.5 \times 10^{6} / \mathrm{ml}\right)$ were plated on poly-L-ornithine-coated $(15 \mu \mathrm{g} / \mathrm{ml}$; Sigma) glass coverslips in 24-well Nunclon $(1.0 \mathrm{ml} /$ well $)$ culture dishes.

Antibodies. Rabbit anti-human $\beta$-galactosidase serum ( $\beta$-gal; used at 1:500 for immunocytochemistry and 1:5000 for Western blot analysis) was purchased from Cappel. Alternatively, a mouse monoclonal anti$\beta$-galactosidase serum (the kind gift of Dr. Alan Peterson, and the Hybridoma Core Facility of the NCE for Neural Regeneration and Recovery, both of McGill University) was used for some studies. Rabbit anti-human neuron-specific enolase serum (NSE; used at 1:2000) was purchased from Polysciences. Mouse monoclonal antibody to human glial fibrillary acidic protein (GFAP; used at 1:100) was purchased from Boehringer Mannheim. Rabbit antiserum to human galactocerebroside (GC; used at 1:200) was purchased from Chemicon. Fluorescein- or rhodamine-conjugated affinipure goat antibodies to mouse or rabbit IgGs were obtained from Jackson.

Immunocytochemistry and quantification. Indirect immunocytochemistry was carried out on cells that were cultured for $1,2,3,6$, and $8 \mathrm{~d}$ in vitro (DIV) on glass coverslips. All coverslips were fixed with $4 \%$ paraformaldehyde for $30 \mathrm{~min}$ followed by three $(10 \mathrm{~min}$ each) washes in phosphate buffered saline (PBS). The cells were then permeabilized with $0.1 \%$ Triton-X in PBS for 5 min followed by three (10 min each) washes in PBS. For dual-labeling experiments the primary antibodies were added together. Following the PBS rinse the coverslips were incubated in the primary antibodies in PBS $/ 10 \%$ normal goal serum for $2 \mathrm{hr}$ at $37^{\circ} \mathrm{C}$. Coverslips were rinsed three times $(10 \mathrm{~min}$ each) in PBS and incubated in secondary antibodies (1:100 for fluorescein-conjugated antibodies and 1:200 for rhodamine-conjugated antibodies) in PBS for $30 \mathrm{~min}$ at $37^{\circ} \mathrm{C}$. Coverslips were then washed three times (10 min each) in PBS, and then rinsed in water, placed on glass slides, and coverslipped with Fluorsave as the mounting medium. Fluorescence was detected and photographed with a Zeiss photomicroscope.

To determine the percentage of cells that were dual labeled with $\beta$-galactosidase and NSE, GFAP, or GC, the cells that were immunopositive for $\beta$-gal were counted first, and then the fluorescent filter was changed to count the cells within that population that were positive for a phenotype marker. The count was random across four or five fields on each coverslip and carried out with a Zeiss photomicroscope. In each 
ERE

-1028 GTATCCAGACTCACTCCTTTCTCCTTACAGTTTCTGAATTCCGTATTAGAAGGGATGGCTCATTTCTAGGGACAAAAT

NF-IL6

- 950 AACACAGGCTCTGGGGTGAGGTAGGGTCGGGTAAGGGGCGAGCGGGGAGGGTGATGGAAGAGCACTACCGCATAAA

VGF/PERIPHERIN

- 872 GAAAGCAAGCTCTCTAGTAAAGCGTTAAGTACTACTTTGTATATTGTTTCTCTTTTTTCCTTTCTCTCTCTCTCTCTC

- $794^{\circ}$ TTTGTTTGTTTTTGGTTTTTGAGACAGAATATCACACTATATATCCAGGCCGGGTACAAAATAACCGCAGTCCTCCGC

- 716 TTCCCAATGCTGGGATTTACAGGCATAAACCACTAAGGGCGGTTATGATGACCTTGAGCTGCAAGTCTTCCTGCCTCT
RARE

E-BOX RARE HOMEODOMAIN

- 638 GCCTCCCAGGTGCTGTTGAGGGTCATAGGCGTGGTCTATTCATACTGAGCTTCTGAATTTGCTCAAATATTAATAATA

E-BOX AP-2 RARE

- 560 ATAGTAATAATAATAATAACCACAATAATACAACTGTAAAACTAAACATTTACCCACGCCTTTTTGACCATCATTCCC

GAMMA - IRE

- 482 ATAGCTCTTGCTACTTTATTTAAAGCGAACAGAGATGTTGAATCCTGACGGAACGTATTTAAATTTÁGTGTAGTATAA

GAMMA - IRE

- 404 ATGAAAAGCTGGAATTTACCATAAAGAATCTCAACACAAATTCTGTGATTAAGTGTTGGGGAAAACTTAAATTATCC

AP - $2 \quad$ bal AP-2

- 326 TAACTACAGTTTAAGGTTAAGCTCCGTATAATCACCCAACCCCCGTTTTCTTTCTTCCCTCTCTACCCCTCCCCAGC

AP - 2

VGF/PERIPHERIN

- 248 TCCACCCCATAATGGATGCTCGGCTAGTTGCTTTTGCGAGGCCTTTGTCTGAAGGATGCAAAATCTACGGATGCTAGC

C/EBP

- 170 GAGGGGGGAAGGGGGGAGAGATTACCTCATACCATGTCGCTTGGACCAATCACCACTCCTGTCGCGGCTTCTCTGGG

PEA-3 AP-2 RARE TATAA

- 92 CAGACGGAGGGTCTGGACCAACAGGAAAAGGCCTTGGCCCATCCCCATGGTGACCGAGCTGTATATAAGGAGGCGCA

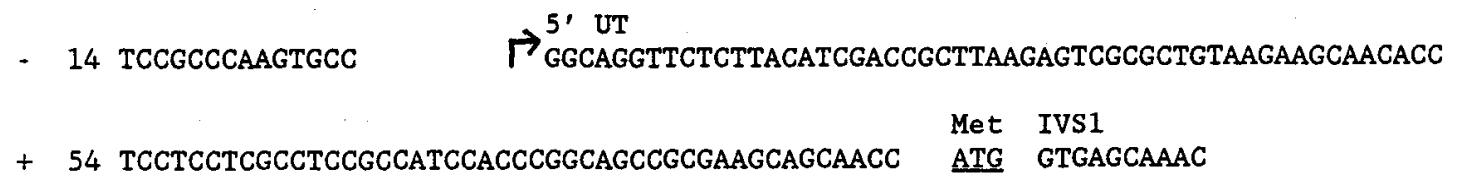

Figure 1. Sequence of the T $\alpha 1 \alpha$-tubulin gene 5 ' flanking region used in the T $\alpha 1$ :nlacZ transgenic mice. The transcriptional start site is indicated by an arrow; the $5^{\prime}$ untranslated region of the mRNA is indicated with $5^{\prime} U T$, and the initiator ATG is underlined. IVSI indicates the start of intron 1. Consensus sequence elements are underlined and identified above the element. In the case of overlapping elements, the second element is in boldface, with identification under the element. In one case where three elements overlapped $(-664)$, the $\gamma$-interferon response element is italicized. ERE, estrogen response element; VGF/PERIPHERIN, sequence that is homologous between the T $\alpha 1$ promoter and these two nervous system-specific genes; $G A M M A-I R E$, $\gamma$-interferon response element; $E-B O X$, bHLH consensus sequence; $R A R E$, retinoic acid response element; HOMEODOMAIN, tandem consensus motifs for Drosophila antennapedia, fushi tarazu, and ultrabithorax homeodomain proteins; $b \alpha 1$, sequence that is conserved between the 5' flanking regions of the T $\alpha 1$ and b $\alpha 1$ genes; $C / E B P$, CCAAT enhancer binding protein. NF-IL6, $A P-2, P E A-3$, and TATAA are self-explanatory (see text).

experiment, at least three coverslips were examined, and this was done in three independent culture experiments.

\section{Results}

Sequence of the T $\alpha 1 \alpha$-tubulin gene 5'flanking region

The transcriptional start site of the T $\alpha 1$ gene, and the DNA sequences immediately $5^{\prime}$ to the start site have previously been characterized (Lemischka and Sharp, 1982). To isolate a genomic clone containing further 5 ' flanking sequences, oligonucleotides homologous to the $5^{\prime}$ untranslated region of the mRNA and to the region immediately $5^{\prime}$ to the start site were used to screen a rat genomic library. Two identical lambda clones were identified, and a $1.9 \mathrm{~kb}$ PstI fragment was subcloned into pGEM-
4Z. This insert contained $1.6 \mathrm{~kb}$ of $5^{\prime}$ genomic flanking sequences, the region corresponding to the $5^{\prime}$ untranslated sequence in the mRNA as well as the translation start site (exon 1), followed by 200 nucleotides of intervening sequences (intron 1). This subclone was used to generate an $\mathrm{SstI} / \mathrm{NcoI}$ partial digestion product that contained 1028 nucleotides of $5^{\prime}$ flanking sequence, the entire $5^{\prime}$ untranslated region ( 99 nucleotides), and the ATG translational start site. This fragment was used to construct a $\mathrm{T} \alpha 1$ :nlacZ fusion gene (see below) for generation of transgenic mice.

The sequence of the $5^{\prime}$ flanking region contained in the fusion gene is shown in Figure 1. As previously reported, this gene has a consensus TATAA box commencing at -30 relative to the 
Figure 2. $\beta$-Galactosidase reporter gene expression in $\mathrm{T} \alpha 1$ :nlacZ transgenic mice. $a-d$, E13.5 embryos from three different lines were stained with X-gal and photographed. $a$ represents the pattern of staining in line $\mathrm{K} 6$, which is the highest-expressing line. $b$ is a dorsal view of an E13.5 embryo from line O12, with the arrow denoting the dorsal root ganglia, which stain intensely with X-gal. $c$ and $d$ are side and frontal views of E13.5 and E14.5 embryos, respectively, from line Q54. The arrow in $c$ indicates the staining that is observed in the gut at this age. Note that in all three lines, expression is detected throughout the nervous system and in the developing eye (the CD1 mice are albinos). $e, \mathrm{An}$ E11.5 embryo from line K6, with the arrow indicating the developing sympathetic chain. Note that at this age, the telencephalon is relatively devoid of transgene expression. $f$, An E10.5 embryo from line Q54, with the arrow indicating the first positively staining dorsal root ganglia.
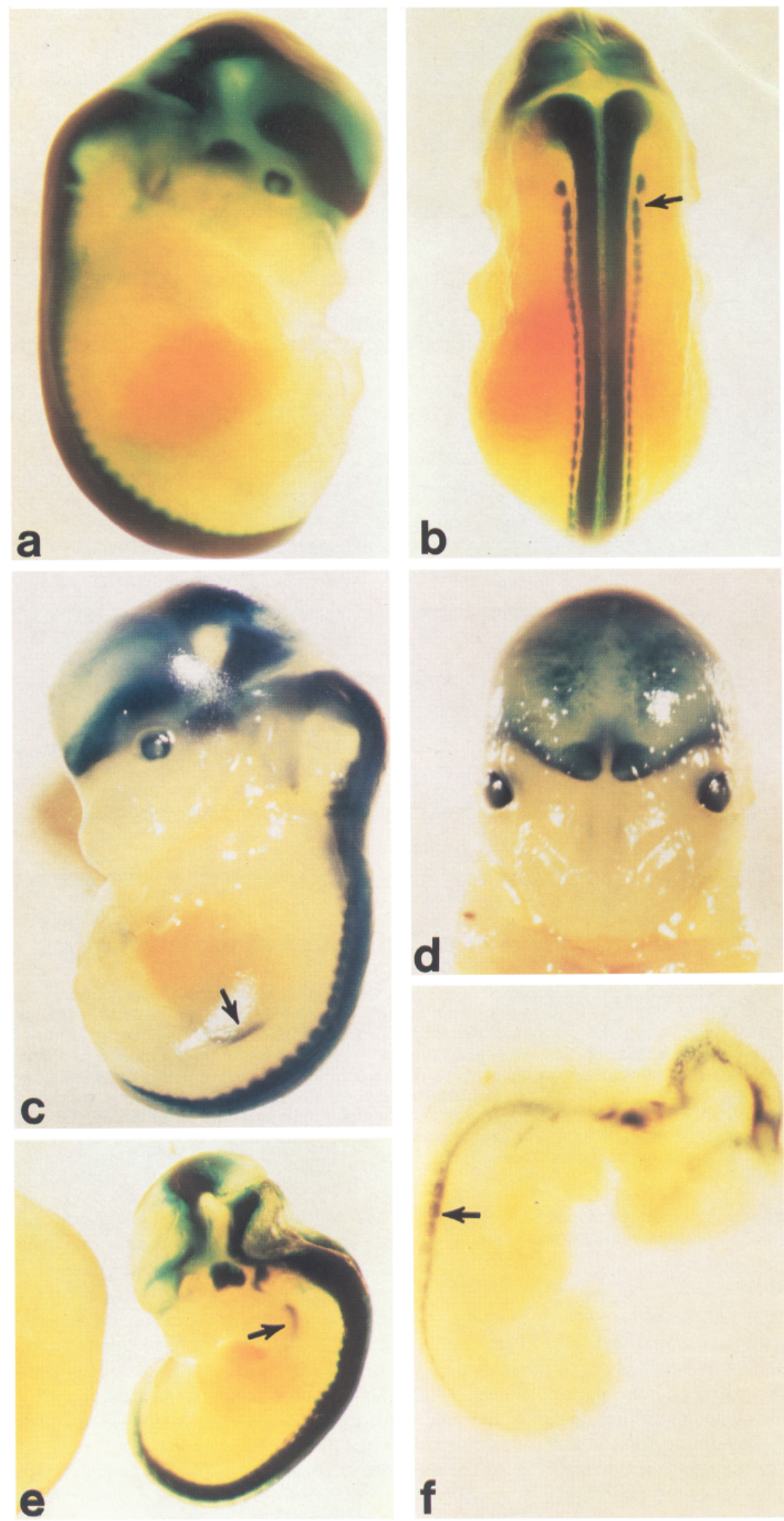
transcriptional start site (Lemischka and Sharp, 1982). Analysis of this sequence revealed a number of consensus sequence elements that might be important both for developmental regulation and for responses to extrinsic cues.

Immediately upstream of the TATAA box are a retinoic acid receptor half-site (Schule et al., 1990), one of four such elements that are located at $-41,-496,-618$, and -669 , and an AP2 site (Mitchell et al., 1987), one of five such elements located at $-54,-214,-260,-291$, and -508 . Other sites that could potentially mediate responses to environmental cues include a PEA-3 site (Wasylyk et al., 1990) at -69, a CCAAT-enhancer binding protein site (Umek et al., 1991) at -143, two imperfect estrogen response elements (Green et al., 1986) at -676 and -959 , three $\gamma$-interferon response elements (Yang et al., 1990) at $-392,-468$, and -664 , and an NF-IL6 consensus element (Faisst and Meyer, 1992) at -899 . These latter two elements are notable because the T $\alpha 1 \alpha$-tubulin gene is induced following neuronal injury (Miller et al., 1989; Tetzlaff et al., 1991).

Commencing at -280 is a region of the $\mathrm{T} \alpha 1$ promoter that shows high homology to the human $\mathrm{b} \alpha 1 \alpha$-tubulin flanking sequences; of 330 upstream nucleotides from this latter gene that have been characterized (Hall and Cowan, 1985), this is the only region of homology. Moreover, embedded within this 19 nucleotide region is a sequence that has been found at approximately the same location in two other neuron-specific, NGFinducible genes, the peripherin (Thompson and Ziff, 1989) and VGF (Salton et al., 1991) genes. Finally, the region between -680 and -490 of the promoter contains a number of elements that are interesting from a developmental perspective. In addition to the aforementioned retinoic acid receptor half-sites (three of them occur within this region), there are two consensus E-box binding sites (Weintraub et al., 1991) for members of the basic helix-loop-helix (bHLH) family (Murre et al., 1989) at -531 and -633 , and two tandem consensus elements from -571 to -541 for the Drosophila homeodomain proteins antennapedia, ultrabithorax, and fushi tarazu (Biggin and Tjian, 1988).

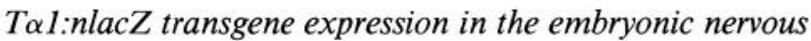 system}

To determine whether $1.1 \mathrm{~kb}$ of $5^{\prime}$ flanking sequences from the $T \alpha 1 \alpha$-tubulin gene was sufficient to direct gene expression to developing neurons, a T $\alpha 1$ :nlacZ fusion gene was made and tested in transgenic mice. The 1127 nucleotide SstI-NcoI partial digest fragment (described above) was fused to an NcoI site located at the ATG translational start site of a modified E. coli lacZ gene derived from pMaori3. This lacZ gene contains an SV40 T-antigen nuclear localization signal inserted in front of the lacZ gene, and has previously been used in transgenic mice (Mercer et al., 1991). A DNA fragment containing the T $\alpha 1$ : nlacZ construct free of vector sequences was isolated and injected into fertilized mouse eggs to generate transgenic founder animals. Transgenic animals were assayed for nlacZ expression by incubating tissues in a staining solution containing $X$-gal, a reagent that leads to deposition of blue crystals in cells with an active $E$. coli $\beta$-galactosidase.

A total of eight lines bearing T $\alpha 1$ :nlacZ transgenes were examined. For all founder lines, embryonic day 13.5 (E13.5) embryos were assessed by histochemical staining with X-gal. At this developmental stage, expression of the endogenous $T \alpha 1$ gene is highly abundant in the embryonic nervous system (Miller et al., 1987). Bacterial $\beta$-galactosidase activity was detected in

\section{9}

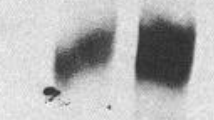

-

Figure 3. Western blot analysis of $\beta$-galactosidase protein in equal amounts of total protein isolated from, 1 , control adult CD1 liver; 2 , line K6 adult liver; 3, line K6 adult neocortex; 4, line K6 P1 neocortex; 5, line Q54 adult liver; 6, line Q54 adult neocortex; 7, line Q54 P1 neocortex; 8, control adult CD1 kidney; and 9, line Q54 adult kidney. The arrow denotes the mobility of $\beta$-galactosidase. Note that only the cortical samples from lines Q54 and K6 contain a $\beta$-galactosidaseimmunoreactive band of the appropriate size, although there is a somewhat larger background band that is present in the control (lanes 1 and 8 ) as well as transgenic (lanes 2 and 9 ) liver and kidney. Note also that $\beta$-galactosidase levels are higher in the adult than in the newborn cortex in both lines of transgenic mice.

embryos derived from five of these lines. Levels of expression of the transgene in these five lines did not correlate with transgene copy number.

In all five positive lines, expression of the transgene was detected only within the developing nervous system (Fig. 2), consistent with the nervous system-specific expression of the endogenous gene in rat embryos. In four of the five lines, Q54, $\mathrm{K} 6, \mathrm{O} 12$, and Q27, expression of the transgene at E13.5 was detected throughout the developing CNS, including the brain, spinal cord, and retina, and, within the PNS, in sympathetic, parasympathetic, and sensory ganglia (Fig. $2 a-d$ ). In the fifth line, line $\mathrm{T} \alpha 1 \mathrm{Z1}$, expression of the transgene at $\mathrm{E} 13$ was detected only in a subset of these sites, including the retina, dorsal root ganglia, and the developing mesencephalon (data not shown); these sites appeared to be those with the highest levels of staining in the other lines.

Two of the lines, Q54 and K6, were chosen for more extensive analysis. The pattern of expression of the T $\alpha 1$ :nlacZ transgene was similar in embryos derived from both lines, although the K6 line expressed higher levels of the transgene, as indicated by the rapidity and intensity of staining with X-gal (Fig. 2a,c). This difference was confirmed by Western blot analysis of the neonatal cortex, which revealed that line K6 expressed much higher levels of $\beta$-galactosidase than did line Q54 (Fig. 3).

To determine precisely the location of the cells expressing the transgene, E13.5 embryos from both lines were stained with $\mathrm{X}$-gal and sectioned. For line Q54, nuclei positive for $\beta$-galactosidase were distributed throughout the embryonic nervous system (Figs. $2 c, 4$ ). $\beta$-Galactosidase-positive cells were detected in the sensory ganglia, including the developing cranial ganglia (Fig. $4 a$ ) and all of the dorsal root ganglia (Figs. $2 c, 4 b$ ). Positive cells were distributed throughout the developing autonomic ner- 
vous system, in both sympathetic ncurons (Fig. $4 c$ ) and parasympathetic neurons of, for example, the gut (Fig. $4 d$ ). Within the CNS, transgene-positive nuclei were detected in the retina, and throughout the spinal cord, myelencephalon, metencephalon, diencephalon, and the developing olfactory lobes (Fig. 2c). At this age, some positive nuclei were detected in the developing neocortex, but $1 \mathrm{~d}$ later, at E14.5, numerous positive cells were detected in the cortical plate region (Fig. $2 d$ ). All of these neuronal populations also express the endogenous $T \alpha 1$ gene at high levels in the E16 rat embryo (Miller et al., 1987).

In line $\mathrm{K} 6$, where transgene expression is many times higher than in Q54, $\beta$-galactosidase staining was detected throughout the E13.5 nervous system in a pattern that appeared to be panneuronal (Fig. 2a). However, at this developmental stage, the $\beta$-galactosidase gene product was not limited to nuclei, presumably because of the very high levels of expression, making mapping of positive cells less definitive than in line Q54.

The endogenous T $\alpha 1 \alpha$-tubulin gene is expressed at the highest levels in those locations within the developing nervous system where neurons are undergoing extensive morphological growth (Miller et al,, 1987). Expression of the transgene was similarly regulated, as exemplified by its expression in the developing retina (Fig. 4e,f). At E16 in the rat, many retinal ganglion cells have been born, have migrated, and are extending thcir axons to form the nascent optic nerve (Horsburgh and Sefton, 1986). At this developmental stage, the endogenous $T \alpha 1$ gene is expressed at high levels within the retina and, more specifically, within the retinal ganglion cell layer, as detected using in situ hybridization (Fig. 4e). At E13.5 in the mouse, the transgene is also expressed at the highest levels in the retinal ganglion cell layer (Fig. 4f). Interestingly, $\beta$-galactosidase-positive nuclei were also noted in the ventricular zone of the developing retina, where retinal precursor cells are dividing, and in the neuroblast layer, where neurogenesis is occurring, and through which the newly committed retinal ganglion cells migrate prior to undergoing differentiation and axogenesis in the retinal ganglion cell layer (Sidman, 1961). These transgene-positive cells might represent either neuroblasts that are committed to a neuronal fate, but are still undergoing division or, alternatively, newly postmitotic neurons prior to their migration from the ventricular zone.

To determine the time of onset of T $\alpha 1$ :nlacZ expression, embryos from lines $\mathrm{K} 6$ and Q54 were examined at earlier time points. At E10.5 (Fig. 2f), $\beta$-galactosidase-positive cells were detected throughout the developing spinal cord and, in the brain, in the myelencephalon, metencephalon, and diencephalon. By E11.5, the level and location of $\beta$-galactosidase expression in the developing CNS were similar to that seen in the E13.5 embryo, with the exception that staining was not yet detected in the retina or in the developing cortical plate (Fig. 2e).

The onset of transgene expression within peripheral ganglia over this time frame was coincident with, and potentially prior to, the birth of sensory and sympathetic neurons. In animals from line Q54, $\beta$-galactosidase-positive nuclei were detected in the region of the coalescing dorsal root ganglia, as soon as the first ganglia were recognizable at E10.5 (Fig. 2f), a time point at which the first neural crest-derived sensory neurons are born (Lawson and Biscoe, 1979). By E11.5, all of the dorsal root ganglia stained intensely for $\beta$-galactosidase (Fig. $2 e$ ). Similar early expression of the transgene was observed in the developing sympathetic chain. In line Q54 at E10.5, $\beta$-galactosidase-positive cells were detected around the dorsal aorta at the level of the superior cervical ganglia, but, at this developmental stage, no ganglionic organization was yet apparent. By E11.5, the superior cervical ganglia were readily distinguishable, and contained numerous $\beta$-galactosidase-positive nuclei. Since the first postmitotic neurons are born at around E12 in the mouse SCG (Hall and Landis, 1991), these correlative data suggest that the transgene may be induced in sympathoblasts prior to terminal mitosis.

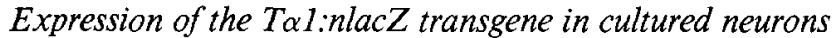 and glial cells}

To determine the identity of the transgene-positive cells that were observed in vivo, primary mixed cultures derived from line K6 were analyzed immunocytochemically. More specifically, mixed cultures derived from whole brains of E14 transgenic embryos were plated onto poly-L-ornithine-coated glass coverslips, and were cultured for $1,2,3,6$, and $8 \mathrm{~d}$ in vitro (DIV). These cultures were then double labeled with antibodies specific for $\beta$-galactosidase, and for one of three different markers: neuron-specific enolase (NSE) for neurons, glial fibrillary acidic protein (GFAP) for astrocytes, and galactocerebroside (GC) for oligodendrocytes.

To determine the identity of transgene-expressing cells in these cultures, $\beta$-galactosidase-positive cells were first identified, and then the filter was changed to determine whether these cells were positive for a second, cell type-specific marker (Fig. 5). In cultures that were double labeled with GFAP, of $1964 \beta$-galactosidase-positive cells that were counted (ranging from 312 to 496 cells at each of $1,2,3,6$, and 8 DIV), none were positive for this astrocytic marker (Fig. $5 C, D$ ). Similarly, in cultures ranging from 1 to 8 DIV that were double labeled with $\mathrm{GC}$, of 576 $\beta$-galactosidase-positive cells that were counted (ranging from 90 to 183 at each time point), none were positive for this oligodendrocyte marker (Fig. $5 E, F$ ). Thus, neither astrocytes nor oligodendrocytes expressed the $\mathrm{T} \alpha \mathrm{l}$ :nlac $\mathrm{Z}$ transgene.

In contrast, at all time points from 1 to $8 \mathrm{DIV}$, a large percentage of $\beta$-galactosidase-positive cells were positive for NSE (Fig. $5 A, B$ ). At $1,2,6$, and $8 \mathrm{DIV}$, of $400-600 \beta$-galactosidasepositive cells counted at each time point, $50-60 \%$ were NSE positive. At $3 \mathrm{DIV}$, the percentage of $\beta$-galactosidase-positive, NSE-positive cells was somewhat lower, bcing approximately $35-40 \%$. Thus, the $\mathrm{T} \alpha 1$ :nlacZ transgene was expressed in neurons. This conclusion was confirmed on the basis of morphology of the $\beta$-galactosidase-positive cells. The identity of the $\beta$-galactosidase-positive cells that were not NSE-positive is somewhat speculative, since they were not, on the basis of the GFAP and GC double-label studies, glial cells. Instead, they may represent early postmitotic neurons that have not yet induced NSE or, in light of the in vivo data, they may represent neuroblasts that have not yet become postmitotic.

\section{Expression of the T $\alpha 1: n l a c Z$ transgene during neuronal maturation and regeneration}

These developmental data indicated that the T $\alpha 1$ :nlacZ transgene was induced parallel with the neuronal commitment decision, and was then expressed, like the endogenous gene, at the highest levels in neuronal populations that were undergoing extensive axonal outgrowth. We have previously demonstrated that $\mathrm{T} \alpha \mathrm{l} \alpha$-tubulin mRNA levels decrease coincident with neuronal maturation (Miller et al., 1987). To determine whether the $\mathrm{T} \alpha 1$ promoter region utilized in the transgene contained the sequences responsible for this developmental downregulation, we examined marker gene expression in the brains of mature 

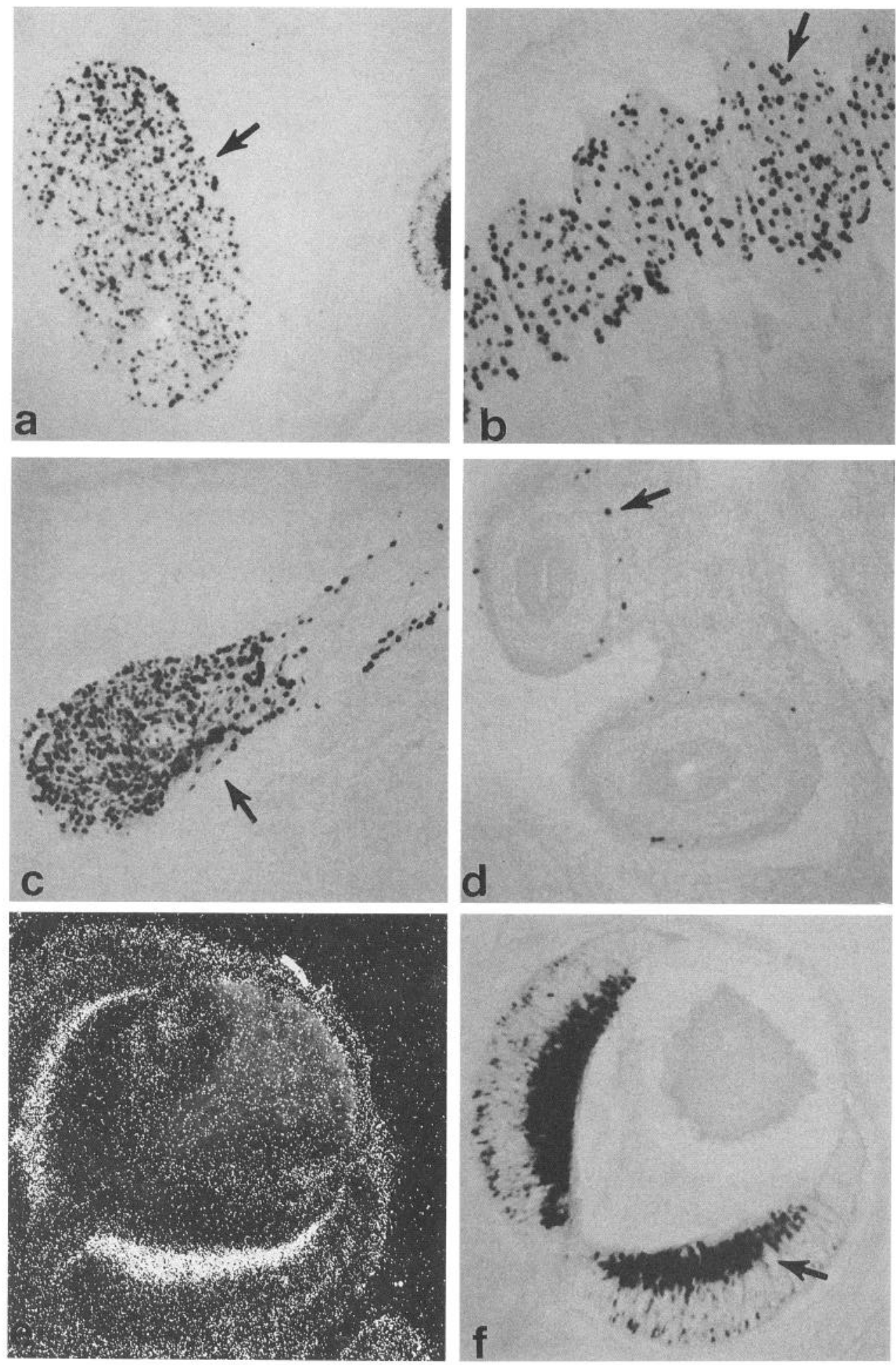

Figure 4. Detection of $\beta$-galactosidase-positive nuclei in sections of E13.5 embryos derived from line Q54. $a$, A horizontal section through the developing head, showing the developing trigeminal ganglion (arrow). $b$, A sagittal section through the dorsal root ganglia (arrow), all of which contain positive nuclei, although the density is lower than that observed in the superior cervical ganglion (c), as would be expected if the positive cells were neurons. $c$, A sagittal section through the developing superior cervical ganglion (arrow) showing numerous positive cells. $d$, A sagittal section through the developing gut, with the arrow denoting positive nuclei that correspond, presumably, to developing parasympathetic neurons. $e$ and $f, \mathrm{~A}$ comparison of the distribution of the endogenous T $\alpha 1 \alpha$-tubulin mRNA in the developing rat eye at E16 (e) versus the T $\alpha 1$ :nlacZ transgene in line Q54 transgenic mice at E13.5 $(f)$. $e$, In a horizontal section of the developing eye, $T \alpha 1$ mRNA levels were highest in the developing retinal ganglion cell layer, as detected using in situ hybridization and emulsion autoradiography. Similarly, in horizontal sections of the transgenic mouse eye $(f)$, positive nuclei were primarily localized to the developing retinal ganglion cell layer (arrow), but a significant number of $\beta$-galactosidase-positive cells were also localized to the ventricular zone (the outermost layer of the retina) and to the neuroblast layer. transgenic animals. X-gal staining of 1-mm-thick brain sections from adult animals of line Q54 (Fig. 6a) revealed that $\beta$-galactosidase expression was significantly downregulated relative to that observed in embryos of the same line (Figs. 2, 4). More specifically, most neurons in the adult Q54 brain (Fig. 6a), spinal cord, and peripheral ganglia (data not shown) did not stain with $\mathrm{X}$-gal, although staining was still detectable in neurons such as those of the hippocampal formation (Fig. 6a), where the endogenous $\mathrm{T} \alpha 1$ gene is expressed at the highest levels in the adult brain (W. Colmers and F. Miller, unpublished observations). In contrast, in line $\mathrm{K} 6$, where $\beta$-galactosidase expression in the embryo was many times higher than in line Q54 (Fig. 3), X-gal staining was still detectable in neurons throughout the adult nervous system (e.g., see Fig. $6 b$ ), in a pattern similar to that observed for the endogenous gene (data not shown).

To confirm, in a more quantitative fashion, that transgene expression was decreased developmentally, we compared $\beta$-galactosidase levels in the neocortex of adult versus newborn $\mathrm{T} \alpha \mathrm{l}$ : 

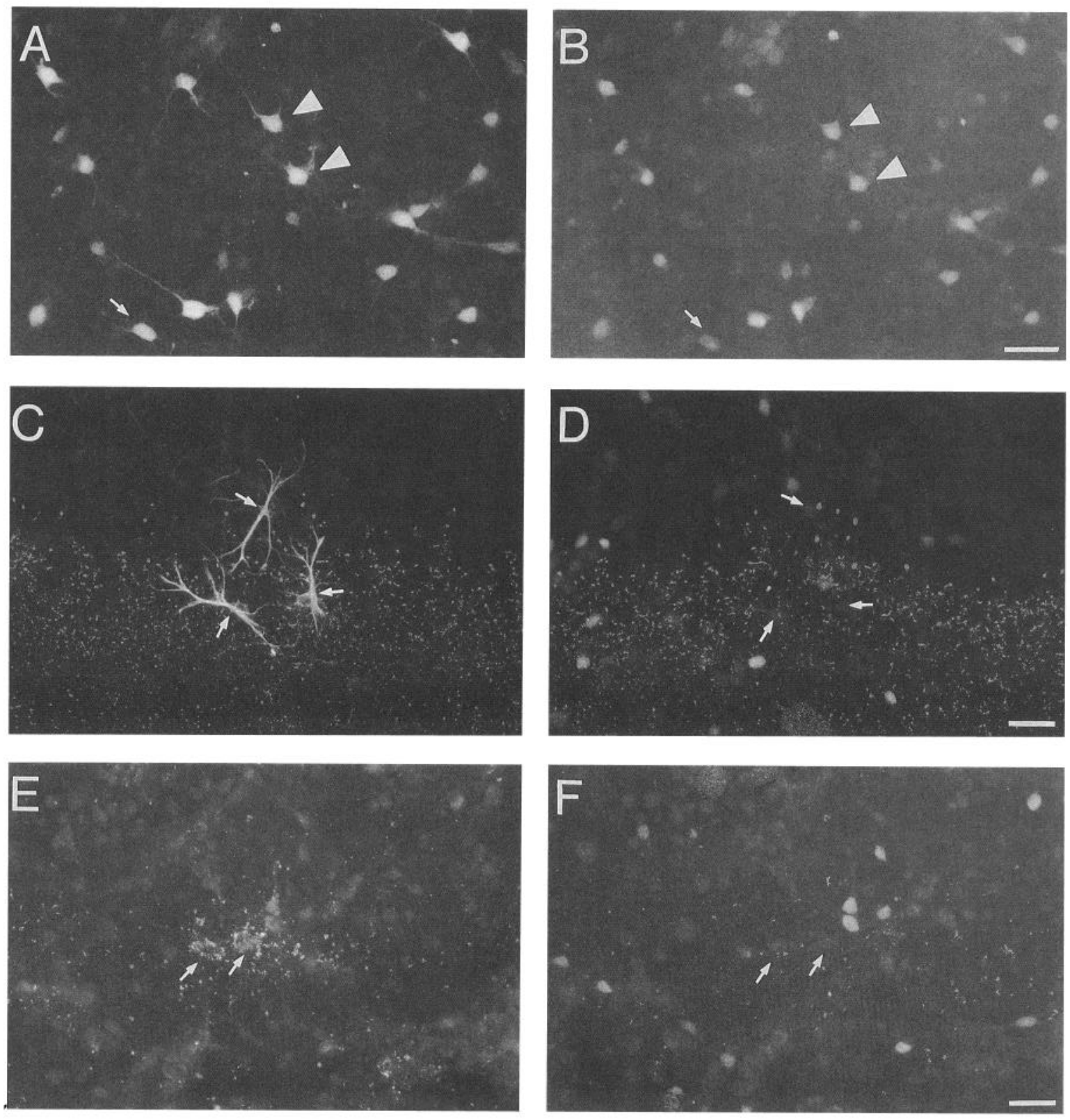

Figure 5. Colocalization of $\beta$-galactosidase immunoreactivity with neuronal but not glial cells in primary mixed cultures derived from transgenic E14 brains of line K6. $A$ and $B$, A majority of the neuron-specific enolase (NSE)-immunoreactive cells $(A)$ also contained $\beta$-galactosidase immunoreactivity $(B)$ (arrowheads), although the occasional NSE-immunoreactive cell was negative for the reporter gene (arrows). $C$ - $F$, Neither astrocytes, labeled with GFAP antibody $(C)$, nor oligodendrocytes, labeled with galactocerebroside antibody $(E)$, indicated by the arrows, contained $\beta$-galactosidase immunoreactivity ( $D$ and $F$, respectively). Scale bars, $50 \mu \mathrm{m}$.

nlacZ mice (Fig. 3). We have previously demonstrated that, in the rat neocortex, over this same time course, endogenous $\mathrm{T} \alpha 1$ $\alpha$-tubulin mRNA levels decrease (Miller et al., 1987). Western blot analysis revealed that, in lines Q54 and K6, transgene expression was downregulated as the neocortex matured (Fig. 3), in agreement with the results obtained from X-gal staining of line Q54. Western blot analysis also confirmed the tissue specificity of transgene expression in the mature animals: detectable $\beta$-galactosidase was present only in the cortex samples, and not in adult liver and kidney of either line (Fig. 3).

To determine whether the transgene was upregulated in mature neurons following axonal injury, as is the endogenous $\mathrm{T} \alpha 1$ mRNA (Miller et al., 1989), we unilaterally crushed or tran- sected the main branch of the facial nerve in adult animals of the K6 line. Five days following axotomy, a time point when the endogenous $\mathrm{T} \alpha 1 \mathrm{mRNA}$ reaches peak levels (Miller et al., 1989), facial motoneurons were assayed for marker gene expression by staining with $\mathrm{X}$-gal. This analysis revealed that staining was detectable in control (Fig. $6 b$ ) facial motoneurons, and that the level of expression was greatly increased in the axotomized motoneurons (Fig. $6 c$ ). Similar results were obtained following nerve crush versus transection, as has been observed for the endogenous gene (Miller et al., 1989). These data therefore demonstrate that the axotomy-induced increase in the T $\alpha 1$ gene expression is mediated, at least partially, at the transcriptional level, and that the elements responsible for this 

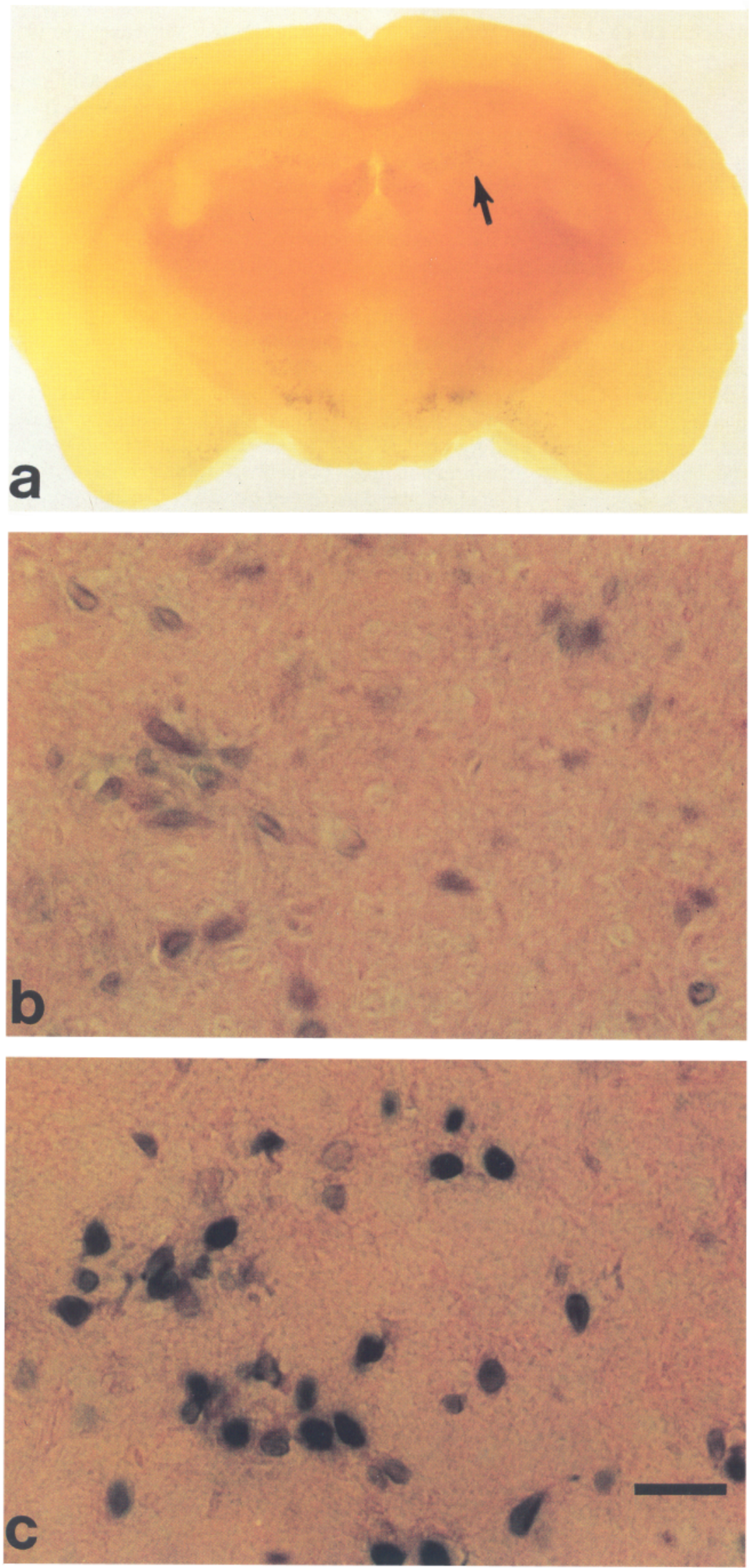

Figure 6. Expression of the T $\alpha 1$ :nlacZ transgene in mature neurons. $a$, Sections 1-2 mm thick of adult brains derived from line Q54 were stained with $\mathrm{X}$-gal overnight and photographed. Note that the majority of the brain was negative for transgene expression, with the exception of a number of neuronal populations where expression of the endogenous T $\alpha 1$ mRNA remains high such as, for example, neurons of the hippocampus (arrow). $b$ and $c$, Expression of $\beta$-galactosidase, as detected by $X$-gal staining and eosin counterstaining, in control $(b)$ and regenerating $(c)$ facial motor neurons from the same section $5 \mathrm{~d}$ following a unilateral crush injury to the main branch of the facial nerve of an adult animal from line $\mathrm{K} 6$. Note that nuclei of the regenerating facial motor neurons $(c)$ are stained significantly more intensely than nuclei from the control, uninjured motor neurons $(b)$. Scale bar (for $b$ and $c$ ), $50 \mu \mathrm{m}$. 
transcriptional increase are present within the $1.1 \mathrm{~kb}$ of promoter region described here.

\section{Discussion}

The endogenous $\mathrm{T} \alpha 1 \alpha$-tubulin gene is expressed at high levels in developing neurons during morphological growth, is downregulated following neuronal maturation, and is then expressed in mature ncurons as a function of extrinsic cucs that regulate growth. In this report, we demonstrate that $1.1 \mathrm{~kb}$ of $5^{\prime}$ flanking region from the $T \alpha 1$ gene is sufficient to specify a similar pattern of gene expression in transgenic mice. More specifically, the $\mathrm{T} \alpha 1$ :nlacZ transgene described here is induced early during neuronal development, and is then regulated as a function of neuronal growth and regeneration. Thus, although we have not ascertained whether this promoter fragment contains all of the DNA sequence elements responsible for regulating relative levels of expression, we can definitively state that it is sufficient to regulate gene expression in a cell type-specific, temporally appropriate fashion.

Data presented in this report indicate that the $\mathrm{T} \alpha 1: \mathrm{nlac} Z$ transgene was induced specifically in neurons early during their development. Although it is difficult to establish definitively a relationship between transgene expression and the birth of neurons using the approaches we describe here, our data do suggest that transgene expression is correlated, in at least some neurons, with terminal mitosis. In the embryonic mouse retina at E13.5 (the stage that we examined), progenitor cells undergo neurogenesis in the neuroblast layer, and subsequently migrate to the ventricular zone to undergo mitosis (Sidman, 1961). Some of these cells migrate back to the neuroblast layer, only to return to the ventricular zone for subsequent mitoses. Others commit to a retinal ganglion cell or amacrine cell fate, and migrate through the neuroblast layer to the retinal ganglion cell layer or to the immediately adjacent region. In this system, we observed transgene-positive cells not only in the retinal ganglion cell and neuroblast layers, but also in the ventricular zone, indicating that the $T \alpha 1: n l a c Z$ transgene was induced either prior to or immediately subsequent to terminal mitosis. Similarly, numerous $\beta$-galactosidase-positive cells were detected in the superior cervical ganglion at E11.5, a time point when sympathetic neurons are just starting to be born (Hall and Landis, 1991). Furthermore, at E10.5, when the ganglion itself was not yet anatomically distinct, transgene-expressing cells, presumably dividing neuroblasts, were detected in the appropriate region adjacent to the dorsal aorta. Precedent for expression of neuronal characteristics in dividing sympathoblasts derives from experiments demonstrating that these cells are catecholaminergic well before they undergo terminal mitosis (Rothman et al., 1978; 1980).

Our in vitro observations are consistent with these in vivo observations; in primary mixed cultures derived from the E14 brain, two populations of $\beta$-galactosidase-positive cells were detected. One population, which comprised approximately $50 \%$ of the total, expressed both $\beta$-galactosidase and neuron-specific enolase and, on the basis of this biochemical marker and their morphology, presumably represented postmitotic neurons. The second population of $\beta$-galactosidase-positive cells, which comprised the other $50 \%$, expressed none of the cell type-specific markers that were used in this study and therefore likely represented neuroblasts that were still dividing and/or neurons that were just postmitotic and that were not yet expressing detectable levels of neuron-specific enolase.
These studies also indicate that, subsequent to its initial induction, the $T \alpha 1: n l a c Z$ transgene is regulated as a function of neuronal growth, as is the endogenous gene (Miller et al., 1987, 1989; Mathew and Miller, 1990). Three observations support this conclusion. First, at E13.5, the transgene was expressed at the highest levels in those neuronal populations that were undergoing extensive axogenesis, such as retinal ganglion cells. Sccond, transgene expression was downregulated in the developing brain as a function of neuronal maturation. Finally, transgene expression was upregulated in mature, regenerating motor neurons. Thus, the T $\alpha 1 \alpha$-tubulin promoter does not simply encode neuronal specificity, but somehow targets high levels of gene expression to those neurons that are elaborating an extensive axonal or dendritic arbor.

Such specification could be a function of intrinsic and/or extrinsic mechanisms. Thus, the T $\alpha 1 \alpha$-tubulin gene might be induced to low basal levels in all neurons, but increased levels of expression might be intrinsically determined either as a function of whether a given neuron will be, for example, a projection neuron versus an interneuron, or alternatively, as a function of cytoskeletal status. At the other extreme, the T $\alpha 1 \alpha$-tubulin promoter might target basal levels of gene expression to all neurons, with absolute levels of expression being entirely dependent upon extrinsic cues within the environment of the neuron, a possibility suggested by studies demonstrating that this gene is regulated by growth factors (Mathew and Miller, 1990; Miller et al., 1991; Ma et al., 1992). However, perhaps the mosi plausible explanation invokes both of these mechanisms: neuronal levels of $\alpha 1$ gene expression may be partially determined as a function of the morphological phenotype of that particular neuron, with absolute levels of expression bcing a function of extrinsic cues like growth factors and cell-cell contact.

Perhaps one of the most intriguing observations reported here is that the $\mathrm{T} \alpha 1$ promoter region is capable of upregulating neuronal gene expression in response to axonal injury. Although the endogenous T $\alpha 1 \mathrm{mRNA}$ is similarly upregulated following axotomy of peripheral (Miller et al., 1989; Mathew and Miller: 1990) and central (Tetzlaff et al., 1991) neurons, this is the firs1 evidence that this upregulation is mediated, at least partially, at the transcriptional level. Although the cellular mechanisms responsible for such a transcriptional upregulation remain largely speculative, we have performed a number of previous studies addressing this issue. First, we have demonstrated that the axotomy-induced increase in T $\alpha 1$ mRNA in mature sympathetic neurons was not simply due to loss of target contact, but was instead a function of the amount of axon that was lost (Mathew and Miller, 1993). Second, we have demonstrated that local cooling of the nerve, which selectively blocked fast axonal transport, but did not induce a peripheral nerve axotomy response (Wu et al., 1994) was sufficient to induce T $\alpha 1$ mRNA in motor neurons (Wu et al., 1993). On the basis of these results, we have hypothesized that induction of the $\mathrm{T} \alpha 1$ gene following axona] injury is due to the loss of ongoing repressive homeostatic signals that derive from the nonterminal axon. Whether the loss of such ongoing cellular signals is translated, at the nuclear level, intc transcriptional induction or derepression remains to be elucidated.

Together, these studies indicate that $1.1 \mathrm{~kb}$ of $5^{\prime}$ flanking sequences from the T $\alpha 1 \alpha$-tubulin gene is sufficient (1) to specify gene expression to neurons early in their development and (2) to regulate levels of gene expression as a function of morphological growth. Although the sequence elements responsible for 
this pattern of genc cxpression are undefined, sequence analysis of the promoter region utilized in this transgene revealed the presence of a number of intriguing motifs. First, this promoter region contains a large number of sequence elements that mediate responses to extrinsic cues. More specifically, this promoter fragment contains five AP-2 sites (Mitchell et al., 1987), a PEA-3 site (Wasylyk et al., 1990), two imperfect estrogen response elements (Green et al., 1986), four retinoic acid halfsites (Schule et al., 1990), an NF-IL6 binding site (Faisst and Meyer, 1992), and three $\gamma$-interferon response elements (Yang et al., 1990). Any of these elements could play an important role in regulating the $T \alpha 1$ gene in response to ongoing environmental cues either during normal development and/or following injury.

In addition to sequence elements that mediate responses to the environment, the T $\alpha 1$ promoter fragment includes two other clusters of sequence motifs that are particularly interesting. One cluster commences at -295 and includes, within 45 nucleotides, two AP-2 sites and a 19 nucleotide region of homology with the promoter of the human b $\alpha 1 \alpha$-tubulin gene (Hall and Cowan, 1985), a region that also bears high homology to a previously noted sequence element found in the $5^{\prime}$ flanking regions of the peripherin and VGF genes (Thompson et al,, 1992). Interestingly, although the peripherin gene is not expressed in all neurons (as is the T $\alpha 1$ gene), it is expressed in a subpopulation of developing neurons early in embryogenesis, is downregulated as a function of neuronal maturation, and is subsequently reinduced during neuronal regeneration (Oblinger et al., 1989; Escurat et al., 1990).

The second cluster of consensus elements occurs from -670 to -490 and includes three of the retinoic acid half-sites, two E-box motifs (consensus elements for members of the bHLH transcription factors), and two tandem consensus sequence elements for a number of Drosophila homeodomain proteins, including antennapedia, ultrabithorax, and fushi tarazu (Biggins and Tjian, 1988). Each one of these three different classes of transcription factors have been implicated during development of the mammalian nervous system (Chisaka et al., 1991; McGinnis and Krumlauf, 1992; Guillemot et al., 1993; Kaplan et al., 1993; Kessel, 1993; Krumlauf ct al., 1993), and this region may therefore play a role in determining the early developmental pattern of T $\alpha 1 \alpha$-tubulin gene expression.

In summary, the T $\alpha 1 \alpha$-tubulin promoter described here is sufficient to specify gene expression coincident with commitment to a neuronal phenotype and to regulate levels of gene expression as a function of neuronal growth and regeneration. Although we have, as of yet, no direct information regarding the sequence elements responsible for determining this pattern of neuronal gene expression, we feel that this issue is now approachable.

\section{References}

Biggin MD, Tjian R (1988) Transcription factors that activate the ultrabithorax promoter in developmentally staged extracts. Cell 53: 699-711.

Chisaka O, Musci TS, Cappecchi MR (1991) Developmental defects of the ear, cranial nerves and hindbrain resulting from targeted disruption of the mouse homeobox gene Hox-1.5. Nature 355:516-520.

Cowan NJ, Cobner PR, Fuchs EV, Cleveland DW (1983) Expression of human $\alpha$-tubulin genes: interspecies conservation of 3' untranslated regions. Mol Cell Biol 3:1738-1745.

Daniels MP (1972) Colchicine inhibition of nerve fiber formation in vitro. J Cell Biol 53:164-176.
Escurat M, Djabali K, Gumpcl M, Gros F, Porticr M-M (1990) Differential expression of two neuronal intermediate-filament proteins peripherin and the low-molecular-mass neurofilament protein (NFL), during the development of the rat. J Neurosci 10:764-784.

Faisst S, Meyer S (1992) Compilation of vertebrate-encoded transcription factors. Nucleic Acids Res 20:3-26.

Ginzburg J, Behar L, Givol D, Littauer UZ (1986) The nucleotide sequence of rat $\alpha$-tubulin: 3 '-end characteristics and evolutionary conservation. Nucleic Acid Res 9:2691-2697.

Green S, Walter P, Kumar V, Krust A, Bornert J-M, Argos P, Chambon $P$ (1986) Human oestrogen receptor cDNA: sequence, expression and homology to v-erb-A. Nature 320:134-139.

Guillemot F, Lo L-C, Johnson JE, Auerbach A, Anderson DJ, Joyner AL (1993) Mammalian achaete-scute homologue 1 is required for the early development of olfactory and autonomic ncurons. Cell 75: 463-476.

Hall AK, Landis SC (1991) Early commitment of precursor cells from rat superior cervical ganglion to neuronal or nonneuronal fates. Neuron $6: 741-752$.

Hall JL, Cowan NJ (1985) Structural features and restricted expression of a human $\alpha$-tubulin gene. Nucleic Acids Res 13:207-223.

Horsburgh GM, Sefton J (1986) The early development of the optic nerve and chiasm in embryonic rat. J Comp Neurol 243:547-560.

Kaplan DR, Matsumoto K, Lucarelli E, Thiele CJ (1993) Induction of trkB by retinoic acid mediates biological responsiveness to BDNF and differentiation of human neuroblastoma cells. Neuron 11:321331.

Kessel M (1993) Reversal of axonal pathways from rhombomere 3 correlates with extra Hox expression domains. Neuron 10:379-393.

Krumlauf R (1993) Hox genes and pattern formation in the branchial region of the vertebrate head. Trends Genet 9:106-112.

Landford RE, White RG, Durnham RG, Kanda P (1988) Effect of basic and nonbasic amino acid substitutions on transport induced by simian virus $40 \mathrm{~T}$-antigen synthetic peptide nuclear transport signals. Mol Cell Biol 8:2722-2729.

Lawson SN, Biscoe TJ (1979) Development of mouse dorsal root ganglion: an autoradiographic and quantitative study. J Neurocytol $8: 265-274$

Lemischka I, Sharp PA (1982) The sequence of an expressed rat $\alpha$-tubulin gene and a pseudogene with an inserted repetitive element. Nature 300:330-335.

Lemischka IR, Farmer S, Racaniello R, Sharp PA (1981) Nucleotide sequence and evolution of a mammalian $\alpha$-tubulin mRNA. J Mol Biol 150:101-120.

Lewis SA, Lee MG-S, Cowan NJ (1985) Five mouse tubulin gene isotypes and their regulated expression during development. J Cell Biol 101:852-861.

Ma Y, Campenot RB, Miller FD (1992) Concentration-dependent regulation of neuronal gene expression by nerve growth factor. J Cell Biol 117:135-141.

Mathew TC, Miller FD (1990) Increased expression of T $\alpha 1 \alpha$-tubulin mRNA during collateral and NGF induced sprouting of sympathetic neurons. Dev Biol 141:84-92.

Mathew TC, Miller FD (1993) Induction of T $\alpha 1 \alpha$-tubulin mRNA during neuronal regeneration is a function of the amount of axon lost. Dev Biol 158:467-474.

McGinnis W, Krumlauf R (1992) Homeobox genes and axial patterning. Cell 68:283-302.

Mercer EH, Hoyle GW, Kapur RP, Brinster RL, Palmiter RP (1991) The dopamine $\beta$-hydroxylase gene promoter directs expression of $E$. coli lacZ to sympathetic neurons in adult transgenic mice. Neuron 7:703-716.

Miller FD, Naus CCG, Durand M, Bloom FE, Milner RJ (1987) Isotypes of $\alpha$-tubulin are differentially regulated during neuronal maturation. J Cell Biol 105:3065-3073.

Miller FD, TetzlaffW, Fawcett JW, Milner RJ (1989) Rapid induction of the major embryonic $\alpha$-tubulin mRNA, T $\alpha 1$, during regeneration in adult rats. $J$ Neurosci 9:1452-1463.

Miller FD, Speelman A, Mathew TC, Fabian J, Chang E, Pozniak C, Toma JG (1994) Nerve growth factor derived from terminals selectively increases the ratio of $\mathrm{p} 75$ to trkA NGF receptors on mature sympathetic neurons. Dev Biol 161:206-217.

Mitchell PI, Wang C, Tjian R (1987) Positive and negative regulation of transcription in vitro: enhancer-binding protein AP-2 is inhibited by SV40 T antigen. Cell 50:847-861. 
Murrc C, McCaw PS, Vacssin H, Caudy M, Jan LY, Jan YN, Cabrera $\mathrm{CV}$, Buskin JN, Hauschka SD, Lassar AB, Weintraub H, Baltimore D (1989) Interactions between heterologous helix-loop-helix proteins generate complexes that bind specifically to a common DNA sequence. Cell 78:537-544.

Oblinger MM, Wong J, Parysek LM (1989) Axotomy-induced changes in the expression of a type III neuronal intermediate filament gene. J Neurosci 9:3766-3775.

Peschon JJ, Behringer RR, Brinster RL, Palmiter RD (1987) Spermatid-specific expression of protamine 1 in transgenic mice. Proc Natl Acad Sci USA 84:5316-5319.

Rothman TP, Gershon MD, Holzer H (1978) The relationship of cell division to the acquisition of adrenergic characteristics by developing sympathetic ganglia cell precursors. Dev Biol 65:322-341.

Rothman TP, Specht LA, Gcrshon MD, Joh TH, Tcitclman G, Pickel VM, Reis DJ (1980) Catecholamine biosynthetic enzymes are expressed in replicating cells of the peripheral but not central nervous system. Proc Natl Acad Sci USA 77:6221-6225.

Salton SRJ, Fischberg DJ, Dong K (1991) Structure of the gene encoding VGF, a nervous system-specific mRNA that is rapidly and selectively induced by nerve growth factor in PC12 cells. Mol Cell Biol 1 1:2335-2349.

Sanger F, Nicklen S, Coulson AF (1977) DNA sequencing with chainterminating inhibitors. Proc Natl Acad Sci USA 74:5463-5467.

Schule R, Umesono K, Mangelsdorf DJ, Bolado J, Pike JW, Evans RM (1990) Jun-Fos and receptors for vitamins $A$ and $D$ recognize a common response element in the human osteocalcin gene. Cell 61: 497-504.

Sidman RL (1961) Histogenesis of the mouse retina studicd with tritiated thymidine. In: The structure of the eye (Smelser GK, ed), pp 487-505. New York: Academic.

Tetzlaff W, Alexander SW, Miller FD, Bisby MA (1991) Response of facial and rubrospinal neurons to axotomy: changes in mRNA expression for cytoskeletal proteins and GAP-43. J Neurosci 11:25282544 .
Thompson MA, Ziff EB (1989) Structure of the gene encoding peripherin, an NGF-regulated neuronal-specific type III intermediate filament protein. Neuron 2:1043-1053.

Thompson MA, Lee E, Lane P, Gizang-Ginsberg E (1992) Nerve growth factor-induced derepression of peripherin gene expression is associated with alterations in proteins binding to a negative regulatory element. Mol Cell Biol 12:2501-2513.

Umek RM, Friedman AD, McKnight SL (1991) CCAAT-enhancer binding protein: a component of a differentiation switch. Science 251 : 288-292.

Villasante A, Wang B, Dobner P, Dolph P, Lewis SA, Cowan NJ (1986) Six mouse $\alpha$-tubulin mRNAs encode five distinct isotypes; testisspecific expression of two sister genes. Mol Cell Biol 6:2409-2419.

Wang D, Villasante A, Lewis SA, Cowan NJ (1986) The mammalian $\beta$-tubulin isotypcs. J Ccll Biol 103:1903-1910.

Wasylyk B, Wasylyk C, Flores P, Begue A, Leprince D, Stehelin D (1990) The c-ets proto-oncogenes encode transcription factors that cooperate with c-Fos and c-Jun for transcriptional activation. Nature 346:191-193.

Weintraub H, Davis R, Tapscott S, Thayer M, Krause M, Benezra R, Blackwell TK, Turner D, Rupp R, Hollenberg S (1991) The myoD gene family: nodal point during specification of the muscle cell lineage. Science 251:761-766.

Wu W, Mathew TC, Miller FD (1993) Evidence that the loss of homeostatic signals induces regeneration-associated alterations in neuronal gene expression. Dev Biol 158:456-466.

Wu W, Toma JG, Chan H, Smith R, Miller FD (1994) Disruption of fast axonal transport in vivo leads to alterations in Schwann cell gene expression. Dev Biol 163:423-439.

Yang Z, Sugawara M, Ponath PD, Wessendorf L, Banerji J, Li Y, Storminger JL (1990) Interferon gamma response region in the promoter of the human DPA gene. Proc Natl Acad Sci USA 87:9226-9230. 